\title{
Simulation of Congenital Heart Defect Corrective Surgeries Using Thin Shell Elements
}

\author{
Stefan Kislinskiy ${ }^{1}$, Tomáš Golembiovský ${ }^{2,3}$, Christian Duriez $^{3}$, Eugénie \\ Riesenkampff $^{4}$, Titus Kuehne ${ }^{4}$, Hans-Peter Meinzer ${ }^{1}$, and Tobias Heimann ${ }^{1,5}$ \\ ${ }^{1}$ German Cancer Research Center (DKFZ), Heidelberg, Germany \\ ${ }^{2}$ Faculty of Informatics, Masaryk University, Brno, Czech Republic \\ ${ }^{3}$ Inria Lille - Nord Europe, Lille, France \\ ${ }^{4}$ German Heart Institute Berlin (DHZB), Berlin, Germany \\ ${ }^{5}$ Siemens AG, Corporate Technology, Erlangen, Germany
}

\begin{abstract}
Congenital heart defect (CHD) corrective interventions in infants are extremely challenging due to commonly complex and heterogeneous disease patterns. At present, cardiac surgeons can only rely on non-invasive imaging prior to surgery. Critical decisions regarding the surgical procedure of choice and its execution must be made during the actual surgery and are strongly dependent on experience. We want to improve surgery planning by providing a simulation system that is able to accurately predict patient-specific results for different surgical procedures preoperatively. Therefore we use a sophisticated simulation model based on thin shell elements. We present a novel joining approach that allows for implementing all necessary surgical low-level procedures, e.g. incising and suturing, independent from the simulation model. No modifications are necessary for already approved thin shell implementations and our simulation system can instantaneously benefit from further improved simulation models in the future. By reducing computationally expensive simulations to a minimum during a virtual surgery we can achieve a fluent workflow for surgeons. However, a specialized mesh resampling algorithm is required to fully utilize our simulation system.
\end{abstract}

\section{Introduction}

Surgical interventions in infants with congenitally malformed great arteries and hearts are extremely challenging due to the complex and heterogeneous nature of their disease patterns. Cardiac surgeons rely on non-invasive imaging for patientspecific examination and preoperatively sketch varying surgical procedures. However, the most promising approach is often chosen during the actual open-heart surgery, when surgeons get a more concrete idea of possible outcomes for different surgical procedures. Finalizing this decision during an on-pump cardiac sur- 
gery is further complicated by deflated blood vessels since it is difficult to imagine their exact change of shape caused by the intervention. Accordingly, important decisions have to be made in very short time and are strongly dependent on the surgeon's experience.

Planning of pediatric cardiac surgeries can be improved by using threedimensional heart models based on patient-specific image data as input for surgical simulation systems. Most present simulation systems focus on execution of low-level surgical procedures, e.g. incising and suturing $[1,2]$. In these reactive simulation systems it is more important to enable simulations in real-time than accurate simulation of tissue and blood vessel deformation in response to simulated interventions.

To be able to predict results of complex surgical procedures a physically more sophisticated simulation is necessary. In [3], a predictive surgical simulation system for bidirectional Glenn shunts and similar surgeries is presented. The authors use a hybrid model to simulate deformations of blood vessels by elastically binding surface meshes to reference Cosserat rods. Although the results are promising, the method is limited to operations on uniform tubular structures and does not allow for arbitrary manipulations of the surface meshes. Suturing of blood vessel walls and patches is accomplished by attaching infinitely contracting springs to split meshes. However, these infinite contraction forces break fundamental physical laws and equations of the simulation model have to be modified accordingly to be able to deal with surplus energy that arises during infinite spring contraction.

In this paper we introduce a novel method for predictive simulation of congenital heart defect (CHD) corrective surgeries which is based on elastic thin shell elements and allows for joining of meshes without using springs. A specific relaxation process during simulation enables our approach to be completely independent from a concrete thin shell elements implementation, i.e. no additional constraints are introduced to the underlying equations. By using SOFA [4], a modular medical simulation framework, we can greatly benefit from this independence as the simulation model can be quickly replaced without any adaption.

\section{Methods}

Finite element methods (FEM) are a well known tool for physically accurate simulations of deformable bodies [5]. Shell elements are two-dimensional finite elements with a certain thickness specified as parameter of the simulation. While they are naturally well-suited for simulation of blood vessel walls and are computationally less expensive than volumetric elements, their computational expense is still higher compared to simple mass-spring methods. However, we achieve a fluent workflow of the surgical planning by reducing the actual simulation to a minimum - instead of executing surgical low-level procedures during simulation, surgeons specify essential properties of the next task before the actual simulation, i.e. both incising and suturing are treated as purely topological manipulations and only 
induced deformations must be simulated. Complex interventions are simulated by consecutive simulation runs while interactions by surgeons occur in between.

Since our method is independent from a concrete simulation model we only provide essential information of our model of choice in the next subsection for the sake of completeness. In-depth descriptions can be found in the referenced literature, i.e. [6,7]. The novel joining approach for meshes, which can be separated into rest shape mapping prior to simulation and rest shape relaxation during simulation, is described subsequently and its limitations regarding possibly necessary mesh preprocessing conclude this section. The whole process is depicted in Fig. 2.1 .

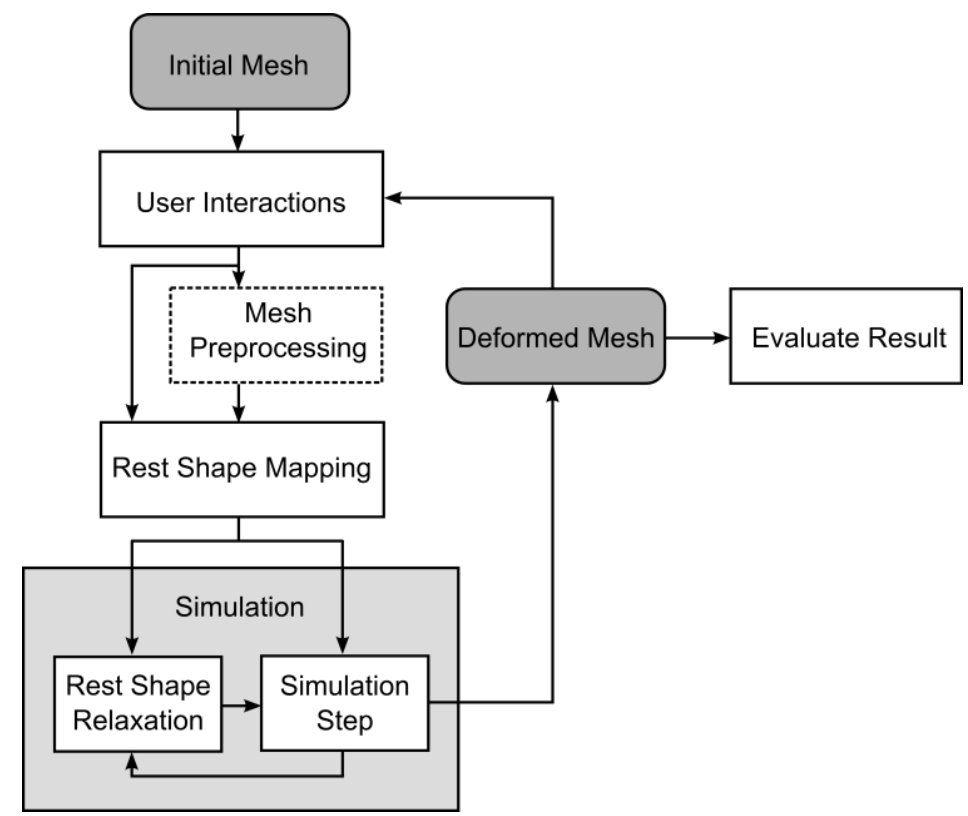

Fig. 2.1 The complete simulation process. Surgeons interact with the mesh prior to simulation by specifying new incisions and edges they wish to join. Especially in case of low shell element count the mesh may be preprocessed to refine and align its vertices along new incisions. Before the actual simulation can start our joining approach requires creation of a rest shape mapping. During simulation, rest shape relaxation is executed in between simulation steps to join split meshes. The deformed mesh may be further manipulated by the surgeon or evaluated in case the simulated surgical procedure is complete.

\subsection{Thin Shell Elements}

Shell elements (see Fig 2.2) combine forces from in-plane deformations, i.e. stretching and shearing, with bending forces. These internal forces $\mathbf{f}$ are a function 
of current displacement measured as difference of the current element shape $\mathbf{x}$ to its rest shape $\mathbf{x}_{0}$.

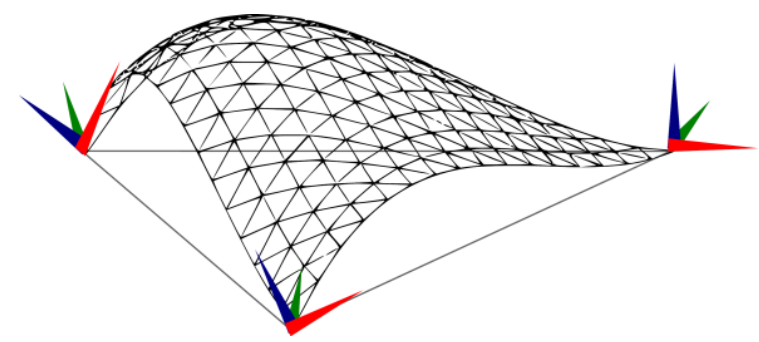

Fig. 2.2 A thin shell element. At each of its 3 nodes 6 degrees of freedom are defined (3 translations, 3 rotations). The shape function of the element allow for smooth interpolation of surface behavior. Shell elements can be bent, twisted, compressed, and stretched.

We accelerate computation by using a corotational formulation that is based on the definition of local frames for each element. In these local frames computation of internal forces is linear:

$$
\mathbf{f}=\mathbf{K}_{e}\left(\mathbf{x}-\mathbf{x}_{0}\right)
$$

where $\mathbf{K}_{\mathrm{e}}$ is the stiffness matrix of the shell element computed by KirchhoffLove's equations. This approach is similar to the model presented in $[6,7]$.

During assembly of simulation meshes, contributions of each shell element are computed in a global frame using different rotation matrices $\mathbf{R}_{\mathrm{e}}$ for each element:

$$
\begin{gathered}
\mathbb{K}=\mathrm{A}_{\mathrm{e}=0}^{\mathrm{N}}\left(\mathbf{R}_{\mathrm{e}}{ }^{\mathrm{T}} \mathbf{K}_{\mathrm{e}} \mathbf{R}_{\mathrm{e}}\right) \\
\mathbb{F}=\mathrm{A}_{\mathrm{e}=0}^{\mathrm{N}}\left(\mathbf{R}_{\mathrm{e}}{ }^{\mathrm{T}} \mathbf{K}_{\mathrm{e}}\left(\mathbf{x}-\mathbf{x}_{0}\right)\right)
\end{gathered}
$$

where A is the assembling operator over $N$ elements, $\mathbb{K}$ is the global stiffness and $\mathbb{F}$ the internal forces of the entire shell element mesh structure. Computation of shell element node motion $\mathbb{U}=\mathbb{K}^{-1} \mathbb{F}$ is performed using a conjugate gradient algorithm.

\subsection{Rest Shape Mapping Prior to Simulation}

We topologically connect the rest shape $\mathbf{x}_{0}$ of an object prior to simulation by joining its vertices along split edges. This connected mesh defines the initial shape $\mathbf{x}$ 
of the simulated object and has fewer vertices as well as a different topology than rest shape $\mathbf{x}_{0}$. Consequently, the 1:n-vertex-mapping needs to be preserved so that adjacent mesh faces which share a vertex can still refer to their distinct vertices of rest shape $\mathbf{x}_{0}$.

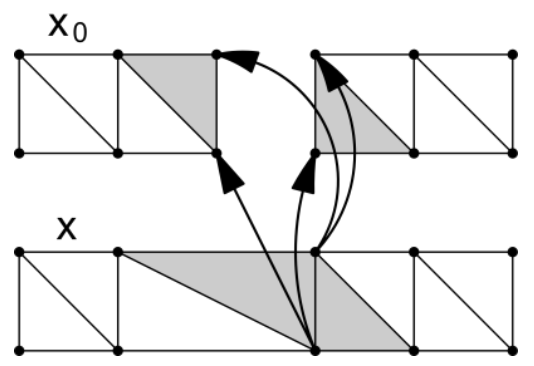

Fig. 2.3 Creation of a topologically joined initial shape $\mathbf{x}$ and the mapping to its rest shape $\mathbf{x}_{0}$ it originates from. The mapping of vertices along the joined edge is explicitly indicated by arrows.

The creation of an initial shape $\mathbf{x}$ and the mapping to its rest shape $\mathbf{x}_{0}$ is shown in Fig. 2.3. For clarity reasons, it represents a minimal scenario (the same concept can be applied to arbitrarily complex real-world scenarios), where two twodimensional elastic stripes are about to be joined. While most of the vertices in the example have a 1:1-mapping, vertices along the split edges have a 1:2-mapping as indicated by arrows in Fig. 2.3. The shaded shell elements share two vertices in initial shape $\mathbf{x}$ and each of them references two distinct vertices of rest shape $\mathbf{x}_{0}$.

\subsection{Rest Shape Relaxation during Simulation}

Depending on the simulated joining scenario, the simulation starts with a large difference between rest shape $\mathbf{x}_{0}$ and the initial shape $\mathbf{x}$. This provokes extremely high internal energies and forces in the affected shell elements. To prevent excessive deformation and numerical instabilities we create a second rest shape $\mathbf{y}_{0}$ which is congruent to the initial shape $\mathbf{x}$ but still has split edges. During simulation we linearly interpolate between the initial rest shape $\mathbf{y}_{0}$ and the original rest shape $\mathbf{x}_{0}$. As soon as rest shape $\mathbf{x}_{0}$ is reached the simulation is in a state where the actual joining is complete and continues until the resulting deformation is complete as well.

The example of Fig. 2.3 is continued in Fig 2.4 to visualize the relaxation process during simulation. The opposite sides of the elastic stripes are fixed so that stretching will occur during simulation. Rest shape and shape of the simulated objects are shown side-by-side for three time steps $t_{0}$ to $t_{2}$. At time $t_{0}$, which represents the very beginning of the simulation, the current rest shape equals $\mathbf{y}_{0}$. It is spatially congruent to shape $\mathbf{x}_{\mathbf{t} 0}$ but differs in topology (this is emphasized in Fig. 
2.4 by contiguous dots at the split edge). At time $t_{1}$ the relaxation of the initial rest shape $\mathbf{y}_{0}$ towards the original rest shape $\mathbf{x}_{0}$ is halfway through. Accordingly, the current rest shape is equally interpolated between rest shapes $\mathbf{y}_{0}$ and $\mathbf{x}_{0}$. Shape $\mathbf{x}_{\mathrm{t} 1}$ converges to its energy equilibrium since it differs spatially from the current rest shape. At time steps $t_{0}$ and $t_{1}$, shapes $\mathbf{x}_{t 0}$ and $\mathbf{x}_{t 1}$ temporarily have no correlation to reality in which the shape would still be split. However, at time $t_{2}$, when the relaxation process is finished, the joining is truly complete.
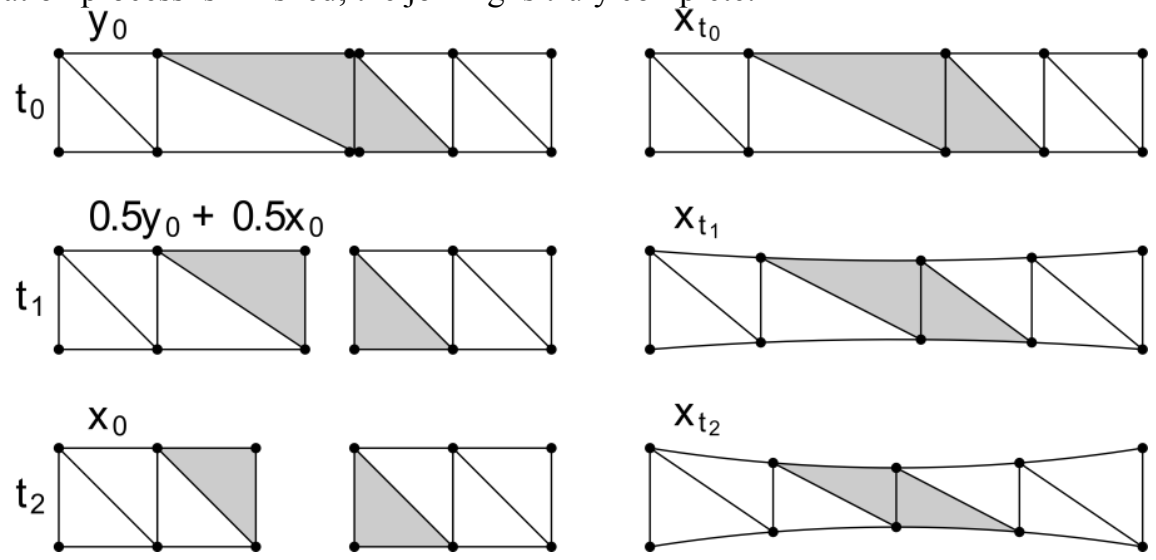

Fig. 2.4 Current rest shape (left) and actual shape (right) are displayed for three time steps $t_{1}$ to $t_{2}$ of the simulation. The stripes are fixed at their opposite ends.

Both rest shape mapping and relaxation can be directly applied to arbitrary complex scenarios. Fig. 2.5 demonstrates the concept during a patch aortoplasty, where a patch is used to widen a coarctation of a blood vessel. While this example is still readily comprehensible, a real world pre-post comparison of a patch aortoplasty is included in Fig. 2.7 besides a subclavian flap aortoplasty and an end-to-end anastomosis. All former examples have in common that the joining requires only a 1:2-mapping. However, our mapping is defined as a 1:n-mapping which allows to combine multiple surgical tasks in a single simulation run. This capability is especially advantageous in case of overlapping incisions which are about to be sutured. Fig. 2.6 shows such a scenario during an end-to-end anastomosis, where one of the blood vessels is widened by a patch to fit the other circumference.

\subsection{Mesh Preprocessing}

As our joining method is solely based on vertices there are important restrictions. On both sides of an incision or edges that are about to be joined there must be the same amount of vertices. To prevent artificial stretching and compression forces along individual joining edges, vertices should be aligned so that all edge pairs 
have similar length each. These constraints have major impact on the resampling of meshes. It is well known that the convergence of finite element methods greatly depends on the quality of the mesh, e. g. regularity of element extent and neighborhood. All these restrictions combined with the requirement of minimizing element count while keeping simulations accurate is a challenging task. The additional requirements originating from our joining approach for a mesh resampling algorithm can be summarized as follows:

- Local alignment of elements along prespecified lines, e.g. incisions.

- Usage of locally prespecified fixed vertices, e.g. along joining edges.

While these constraints are hard constraints at their exact locations, there must be smooth transitions of element alignment and size between them to still produce high quality simulation meshes. For our simulation system prototype (see Fig. 2.7) we currently elude mesh resampling completely by using relatively dense meshes and by snapping incisions along element edges. Joining is restricted on split edges with the same amount of vertices.
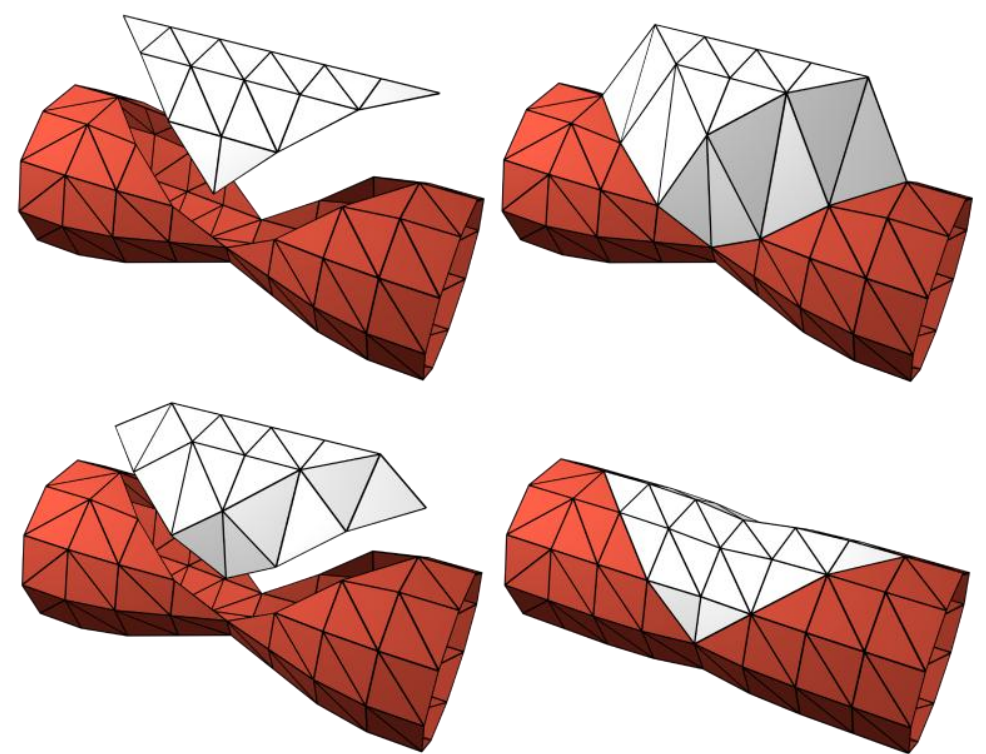

Fig. 2.5 Joining during a patch aortoplasty. From top left to bottom right: rest shape $\mathbf{x}_{0}$, initial shape $\mathbf{x} /$ rest shape $\mathbf{y}_{0}$ (split edges), rest shape during relaxation process, and final shape after simulation. 


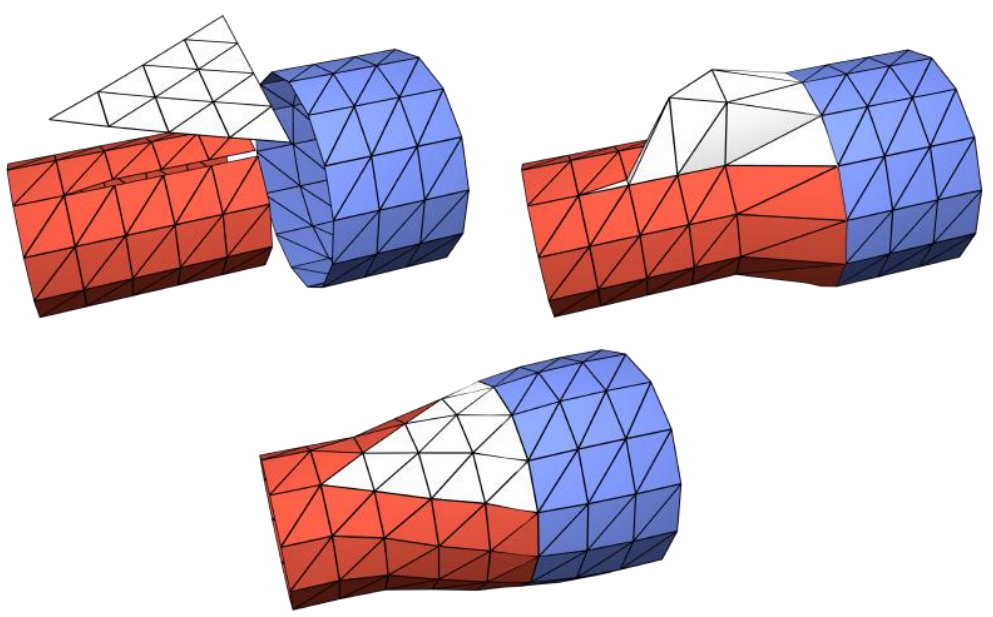

Fig. 2.6 Joining during an end-to-end anastomosis. The circumference of one blood vessel must be widened by a triangular patch. Vertices that are shared between both blood vessels and the patch have a 1:3-mapping to the rest shape. From top left to bottom: rest shape $\mathbf{x}_{0}$, initial shape $\mathbf{x}$ $/$ rest shape $\mathbf{y}_{0}$ (split edges), and final shape after simulation.
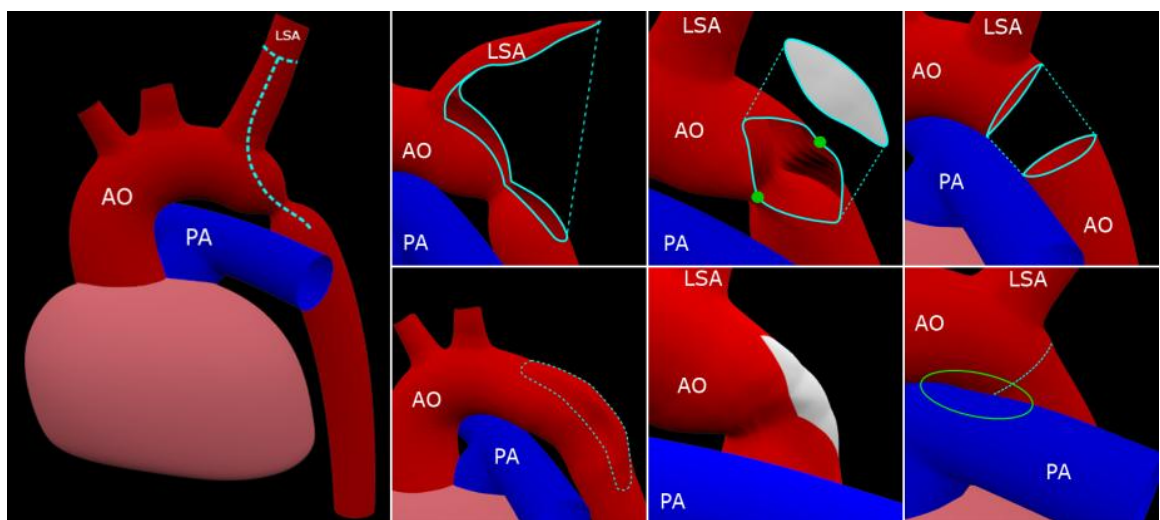

Fig. 2.7 Screenshots of our simulation system prototype for different surgical procedures used to repair a coarctation of an aortic arch. For all scenarios our novel joining approach was used. The screenshots are enriched with overlay delineations for clarification. Left image: Overview of the simulation scene consisting of an aortic arch (AO), the left subclavian artery (LSA) and the pulmonary artery (PA). The coarctation can be seen next to the LSA and the dashed line indicates where the aorta will be incised for a subclavian flap aortoplasty. The vertical image pairs display the scene before and after different surgical interventions. Left image pair: Subclavian flap aortoplasty. The subclavian flap is used as an organic patch to widen the coarctation. Center image pair: Patch aortoplasty. A patch is sutured into an incision along the coarctation. The green dots represent springs that were attached to widen the incision for visualization purposes. Right image pair: End-to-end anastomosis. The coarctation is resected and the loose ends of the $\mathrm{AO}$ are sutured together. Due to deformation of the AO it slightly collides with the PA which causes additional deformations on both blood vessels. 


\section{Results and Discussion}

In order to show an improvement on existing simulation systems for CHD corrective surgeries we want to demonstrate that our blood vessel model based on thin shell elements

- physically meets the requirements for predictive simulation results,

- converges to these results with a moderate number of elements,

- is able to simulate surgical procedures in practice.

We close this section with a discussion about the constraints of our method compared to present simulation systems.

\subsection{Predictive Simulation Results}

A quantitative validation by means of a comparison to real pre- and postsurgery image data is impossible at present. This is mainly due to ethical issues that arise during image acquirement of infants, i.e. necessary heart rate lowering medications or exposure to radiation. To our knowledge there is no image data available that is obtained soon enough after relevant interventions in infants, where the growth of the patient does not interfere comparison. However, a qualitative comparison to real image data acquired months after a surgical intervention, which confirms at least a principal applicability of predictive simulations for surgery planning, can be found in [3].

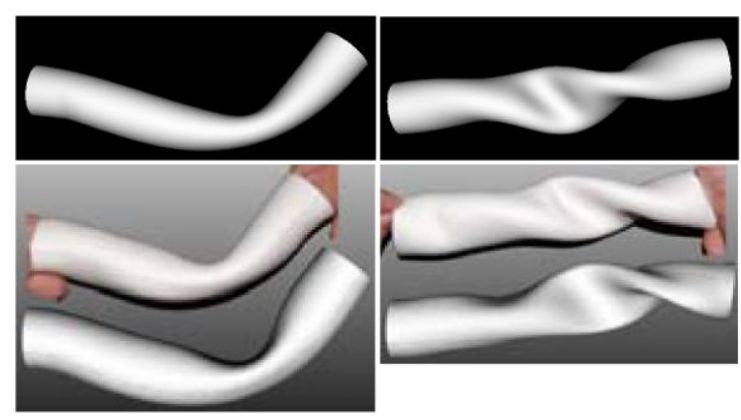

Fig. 3.1 Simulation of bending and twisting of an elastic tube. Top row: Our thin shell element model. Bottom row: Real elastic tube manipulated by hand and Cosserat rod based hybrid model [3] (credits for the bottom images: Li et al.).

At present we can only provide a rudimentary qualitative validation shown in Fig. 3.1, where bending and twisting of an elastic tube manipulated by hand is 
compared to the simulation results of [3] and our shell model. Both deformations are likely to occur during surgery and our results are visually very close to the real deformations as expected due to the physically formulation of shell elements. Minor differences in shape are probably owed to the lack of knowledge of material properties and thickness of the reference model.

\subsection{Convergence of Thin Shell Elements}

We set up an evaluation for the convergence of shell elements for strong deformations of blood vessels in order to gather valuable hints for reasonable mesh resampling while keeping appropriate simulation accuracy (see Fig. 3.2). Therefore we simulated the same deformation on the same mesh sampled at different levels of detail and measured the Hausdorff distance between them. We continuously increased the element count during mesh sampling until the Hausdorff distance between simulation results was negligible. This simulation result was taken as reference and all Hausdorff distances were finally computed against it. To minimize the measuring error we mapped each simulation result to the same dense mesh which in turn was used for comparison.

Although the results of the evaluation can only be used to illustrate tendencies, it is remarkable that despite the strong deformation its shape is preserved very well even for a reduction of the shell element count to $25 \%$ of the reference. While the mean distance is generally low, the maximal distances always occur at locally high curvature, which is expected behavior since the high rate of change in curvature cannot be mapped by the shape functions of relatively large shell elements. This effect can be clearly seen at the bottommost mesh in Fig. 3.2

\subsection{Simulation of Virtual Surgeries in Practice}

As mentioned in Section 2.4 we implemented a basic prototype of our simulation system which is shown in Fig. 2.7 for different surgical procedures. While its usability is restricted due to a missing mesh resampling algorithm, it is convenient for a proof of concept. We created a three-dimensional model of a malformed aortic arch based on real image data and simulated three fundamentally different surgical approaches to repair the coarctation. Especially the subclavian flap aortoplasty is a complex intervention which heavily changes topology. However, it makes no difference for our novel joining approach which is universally applicable for arbitrarily split meshes. 


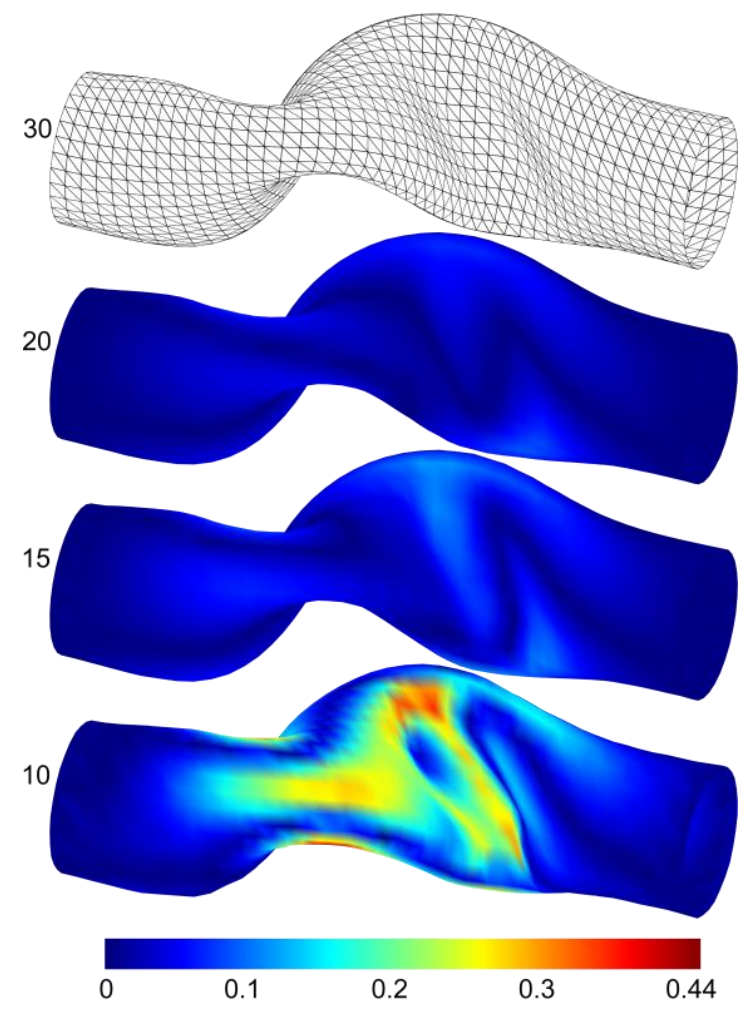

Fig. 3.2 Hausdorff distances between a strongly deformed reference mesh and meshes with lower levels of detail $(20,15$, and 10 vertices along the circumference of the tube compared to 30 vertices). Relative shell element count from top to bottom: $100 \%, 44 \%, 25 \%, 11 \%$. The tube has radius 1 and length 10 .

\subsection{Limitations}

Although our novel joining method can be universally used for joining of arbitrarily split meshes, it enforces special requirements on a suitable mesh resampling algorithm (see Section 2.4). However, simulation systems based on joining by contracting springs must include mesh resampling as well in case a surgeon wants to continue an intervention after joining, whereas there are no additional constraints for the resampling.

Regarding the speed of shell element based simulations we observed an inversely proportional dependency to the shell element count. At approximately 
1000 shell elements, simulation time of our reference implementation elapses faster than real time on average consumer-level hardware. Considering our observations of convergence in Section 3.2, a fast yet predictive simulation system should be feasible with today's hardware and an optimized implementation of a shell element model.

\section{Conclusion}

We presented a modular and independent method for joining meshes in the context of an efficient surgical simulation system for CHD corrective surgeries. There are two major advantages of our approach: Firstly, simulations of virtual surgeries are accelerated despite the usage of a sophisticated simulation model which enables a fluent workflow for surgeons. Secondly, the loose coupling between lowlevel surgical procedures and the simulation model allows for instantaneous utilization of alternative or newly developed and improved shell element implementations in the future. Furthermore, the modularity of our method eases its combination with other simulation models, e.g. volumetric finite element models for heart simulations, especially in the environment of SOFA, an open source framework for medical simulations. However, our simulation system can only be fully utilized in case a specialized mesh resampling algorithm is provided. Development of such an algorithm is consequently the next step towards applicability of our methods in clinical workflow.

\section{References}

1. Mosegaard J (2004) LR-spring mass model for cardiac surgical simulation. MMVR 256-258

2. Sørensen TS, Greil GF, Hansen OK, Mosegaard J (2006) Surgical simulation - a new tool to evaluate surgical incisions in congenital heart disease? Interactive CardioVascular and Thoracic Surgery 5:536-539

3. Li H, Leow WK, Chiu IS (2009) Predictive simulation of bidirectional Glenn shunt using a hybrid blood vessel model. MICCAI 5762:266-274

4. Allard J, Cotin S, Faure F, Bensoussan PJ, Poyer F, Duriez C, Delingette H, Grisoni L (2007) SOFA - An Open Source Framework for Medical Simulation. MMVR 15

5. Reddy JN (1993) Introduction to the Finite Element Method. McGraw-Hill, New York

6. Comas O, Cotin S, Duriez S (2010) A shell model for real-time simulation of intraocular implant deployment. Biomedical Simulation 5958:160-170

7. Comas O, Duriez C, Cotin S (2010) Shell model for reconstruction and real-time simulation of thin anatomical structures. MICCAI 6362:371-379 